\title{
Efecto del extracto de ajo como compensador de frio en la brotación del manzano red delicious en la región de nuevo ideal durango México
}

\author{
Effect of garlic extract as a cold compensator in the spread of the apple \\ red delicious in t nuevo ideal durango, region
}

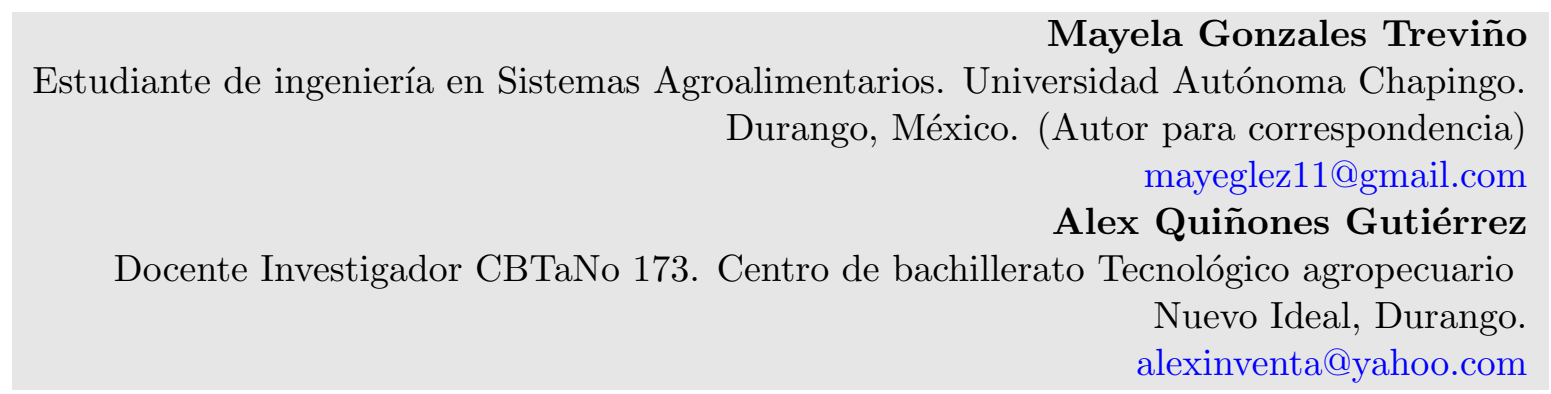

\section{Resumen}

La investigación evaluó los efectos de compensadores de frio orgánicos y químicos en la floración, calidad de fruto y productividad. El diseño experimental fue bloques al azar con 4 tratamientos y 5 repeticiones. T0=control; T1=ajo $\left(5 \mathrm{~kg} 1000 \mathrm{~L}^{-1}+\right.$ glicerina $\left.2 \mathrm{~L} 1000 \mathrm{~L}^{-1}\right)$; T2=Erger $($ (Nitrógeno orgánico $(1 \%)$, nitrógeno inorgánico (nítrico 5\% y amoniacal 5\%) y 50\% de carbono orgánico + nitrato de calcio 5 kg 1000 $\mathrm{L}^{-1}$; T3=Revent $\mathbb{R}$ (Thidiazuron $200 \mathrm{ml} 1000 \mathrm{~L}^{-1}+$ aceite mineral invernal $10 \mathrm{~L} / 100$ ). Se identificaron y marcaron 5 ramas similares, 4 de acuerdo a los puntos cardinales y una central. Se evaluó el número de flores, en frutos el diámetro ecuatorial y polar con un vernier, el peso se determinó con balanza de precisión y porcentaje de coloración, para medir la dureza un penetrómetro, los sólidos solubles con un refractómetro. El análisis de varianza y la prueba de comparación de medias (Tukey; $\alpha=0.05$ ) entre las variables respuesta, se realizó con el paquete estadístico del Dr. Olivares de FAUANL. Hubo diferencias significativas en la floración de los tratamientos $\mathrm{T} 1=178, \mathrm{~T} 2=171$, T3=208 respecto al control T0=91. El diámetro polar y ecuatorial del fruto el T2 fue diferente a los demás (19.7\%). La concentración de sólidos solubles y la dureza de la pulpa no presentaron diferencias, contrario a la coloración el T1 fue diferente a los otros tratamientos (74.78\%). En conclusión, el ajo puede funcionar como compensador de frio en el manzano Red deliciuos, sin embargo, es menos productivo en comparación con los tratamientos químicos utilizados.

Palabras clave: evaluación; compensador; penetrómetro; vernier; biopreparado; residualidad 


\begin{abstract}
The research evaluated the effects of organic and chemical cold compensators on flowering, fruit quality and productivity. The experimental design was random blocks with 4 treatments and 5 repetitions. T0 $=$ control; $\mathrm{T} 1=$ garlic $(5 \mathrm{~kg} / 1000 \mathrm{l}+$ glycerin $211000 \mathrm{l}) ; \mathrm{T} 2=$ Erger $\AA$ (Organic nitrogen $(1 \%)$, inorganic nitrogen (5\% nitric and 5\% ammoniacal) and 50\% organic carbon + calcium nitrate $5 \mathrm{~kg} / 1000$ L; T3 = Revent $\AA$ (Thidiazuron $200 \mathrm{ml} / 10001$ + winter mineral oil $10 \mathrm{l} / 100$ ) Five similar branches were identified and marked, 4 according to the cardinal points and one central.The number of flowers was evaluated, in fruits the equatorial and polar diameter with a vernier, the weight was determined with precision balance and percentage of coloration, to measure the hardness of a penetrometer, the soluble solids with a refractometer The analysis of variance and the comparison test of means (Tukey; $\alpha=0.05$ ) between the response variables, was performed with the statistical package of Dr. Olivares of FAUANL There were significant differences in the flowering of the treatments $\mathrm{T} 1=178, \mathrm{~T} 2=171$, T3 $=208$ with respect to the control $\mathrm{T} 0=91$. The polar and equatorial diameter of the fruit $\mathrm{T} 2$ was different from the rest (19.7\%) The concentration of soluble solids and the hardness of the pulp did not present differences, contrary to the coloration the T1 was different to the other treatments $(74.78 \%)$. In conclusion, garlic can function as a cold compensator in the Red deliciuos apple tree, however, it is less productive compared to the chemical treatments used.
\end{abstract}

Keywords: evaluation; compensator; penetrometer; vernier; biopreparado; residualidad

\section{Introducción}

El uso de químicos es muy común en los agricultores el manejo de cultivos la debida aplicación es la medida más efectiva para aumentar la productividad y mejorar la calidad en los cultivos debido a esto la industria de los agroquímicos ha aumentado los efectos negativos que derivan en el desequilibrio de los ecosistemas. Independientemente del grado de exposición de la población a dichos efectos, la contaminación ambiental por medio de los productos desintegrados en el suelo, agua y aire, son descomunales lo que afecta directamente al medio ambiente y al hombre (Martínez, et al., 2007).

La agricultura orgánica tiene como objetivo garantizar la sustentabilidad, utilizando eficazmente los recursos naturales disponibles en el predio sin hacer uso de químicos o productos sintéticos genéticamente modificados, centrándose en evitar la erosión y conservando la fertilidad del suelo y siendo amigable con el ambiente y la biodiversidad, así como la salud de los agricultores y consumidores (Hage, et al., 2003; Bomcam, 2003).

Según García, et al., (2010), los extractos vegetales contienen distintos componentes químicos que presentan distintas propiedades biológicas que benefician a en distinta manera a otros seres vivos.

Los biopreparados son compuestos a base de materia natural disponible, cuyo fin es proteger y mejorar al medio donde se aplican (Boncam, 2003).

El alto uso inorgánicos traen consigo efectos negativos en el cultivo, por la residualidad, perdidas ecónomas y entre otras (Nava, et al., 2012).

Los frutales caducifolios requieren determinadas horas frio para tener una floración y brotación vegetativa uniforme y vigorosa, en algunas ocasiones dichos requerimientos no se cumplen por la diversidad de clima y las horas frio no se acumulan lo suficiente. en este caso un compensador de frio es un auxiliar que garantiza que las yemas broten de manera homogénea (Feicán, et al., 1998).

El manzano demanda al menos de 1,000 horas frio, dependiendo de la variedad, al no acumularse el requerimiento tiende a afectar la calidad del producto e incluso evita su formación y el correcto desarrollo (Infoagro, 2007). 
En la región de Nuevo Ideal, Dgo. Se acumulan de 350 a 600 horas frio en el periodo invernal por lo que se necesita compensar el faltante de 700 a 1200 horas frio que requiere el manzano por medios químicos u orgánicos para que los árboles se desarrollen un crecimiento vegetativo optimo y obtener frutos de calidad.

El objetivo de la investigación fue evaluar los efectos de los diferentes compensadores tanto orgánico como inorgánicos sobre la dinámica de floración, calidad de fruto y productividad en la variedad Red delicious en el municipio de nuevo Ideal Durango México.

\section{Materiales y métodos}

La investigación se realizó en el Centro de Bachillerato Tecnológico agropecuario de ubicado en el municipio de Nuevo Ideal, Dgo. El cual cuenta con una altitud de 1920 msnm aproximadamente, se localiza en el paralelo $20^{\circ} 53^{\prime \prime}$ latitud norte y en el meridiano $105^{\circ} 44^{\prime \prime}$ de longitud oeste. su clima se caracteriza por ser semiseco templado (Instituto Nacional de Estadística, Geografía e Informática, 1998).

\subsection{Diseño del experimento}

El diseño experimental fue bloques al azar con 4 tratamientos y 5 repeticiones. T0=testigo; $\mathrm{T} 1=$ ajo $(5 \mathrm{~kg} / 1000 \mathrm{l}+$ glicerina $2 \mathrm{~L} / 1000 \mathrm{~L})$; T2=Erger ß (Nitrógeno orgánico (1\%), nitrógeno inorgánico (nítrico $5 \%$ y amoniacal $5 \%$ ) y $50 \%$ de carbono orgánico + nitrato de calcio $5 \mathrm{~kg} 1000 \mathrm{~L}^{-1}$; $\mathrm{T} 3=$ Revent (R) (Thidiazuron $200 \mathrm{~mL} 1000 \mathrm{~L}^{-1}+$ aceite mineral invernal $10 \mathrm{~L} / 100 \mathrm{~L})$. En cada árbol se identificaron y marcaron 5 ramas de similares características, 4 de acuerdo a los puntos cardinales (norte, sur, este y oeste) y una central. Para la realización del experimento se utilizaron un total de 60 árboles de similares características de la variedad Red delicious.

\subsubsection{Preparación del ajo}

Primero se pesaron 1.25 kilogramos de ajo para obtener 250 L de solución posteriormente se retiraron las cascaras de los ajos y luego en mortero se maceraron y se agregó 1.25 litros de alcohol de $96^{\circ}$ y para posteriormente dejar fermentar por 72 horas, con ayuda de un colador limpio el extracto de ajo y finalmente se agregaron $250 \mathrm{~mL}$ de glicerina.

\subsection{Aplicación de los compensadores}

En cada árbol se identificaron y marcaron 5 ramas de similares características, 4 de acuerdo a los puntos cardinales (norte, sur, este y oeste) y una central. para la Aplicación los de compensadores se utilizó un aspersor cuando se observó el $50 \%$ de las yemas florales están en la etapa de punta rosada.

\subsection{Monitoreo}

se contaron las flores de cada una de las ramas seleccionadas cuando la floración se encontraba en un $90 \%$, además de realizar el conteo de frutos amarrados y ejecutar un muestreo superficial de los frutos.

\subsection{Evaluación de los compensadores}

Para evaluar los tratamientos los árboles se marcaron e identificaron con listones de diferente color.

Primero se cortaron los frutos de los arboles tratados y a las manzanas de la muestra se le midió dureza (penetrómetro $\mathrm{Kg} / \mathrm{cm} 2$ ) y azucares solubles (refractómetro) para determinar índices de calidad. Además, se midió tamaño (calibre polar y ecuatorial con un vernier) y el peso (balanza de precisión) para determinar índices de calidad.

\subsection{Análisis de datos}

El análisis de varianza y la prueba de comparación de medias (Tukey; $\alpha=0.05$ ) entre las variables respuesta (Steel \& Torrie, 1986), se realizó con el paquete estadístico del Dr. Olivares (1995) de FAUANL. 


\section{Resultados y discusión}

Se identificaron y marcaron 5 ramas similares de los 60 árboles seleccionados. Se evaluó el número de flores, en frutos el diámetro ecuatorial y polar, el peso, el porcentaje de coloración, la dureza y los sólidos solubles.

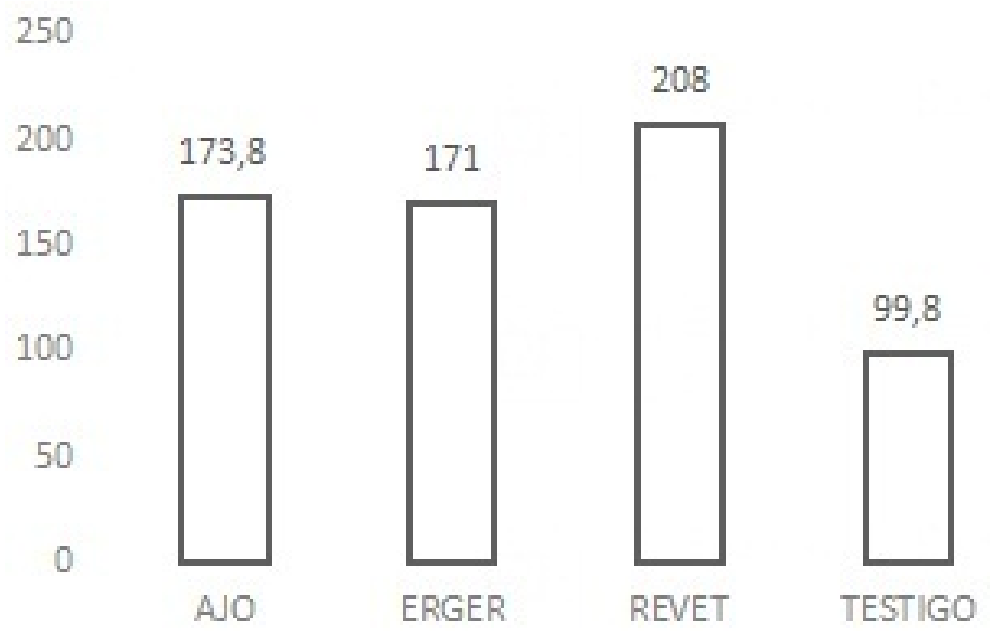

Grafica 1: Floración de las ramas por tratamiento aplicado

Se encontraron diferencias significativas en la mayor incidencia floral, nótese que el testigo floración (gráfica 1) de los tratamientos, mostrando se encuentra muy por debajo de los demás superioridad el tratamiento 3 (Revent) que presento tratamientos.

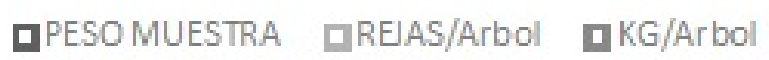

70

60

$$
50
$$

40

30

20

10

0

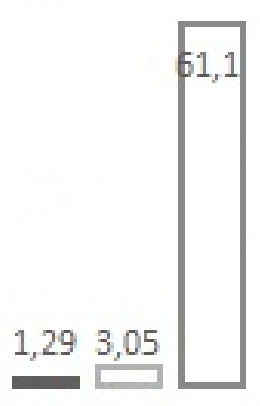

ERGER

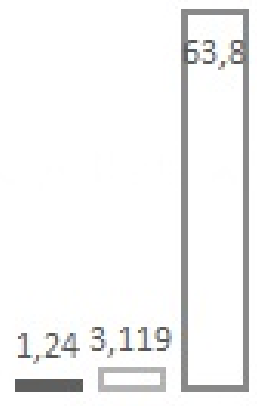

REVET

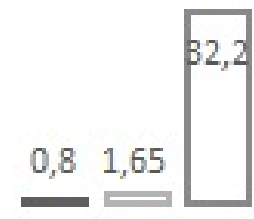

TESTIGO

Grafica 2: Productividad por cada tratamiento aplicado

La productividad de los tratamientos (Gráfica 2) químicos utilizados en el experimento es mayor, ya que los arboles a los que se les aplicaron los tratamientos Revent y Erger, el producto cuenta con un peso superior, y el número de manzanas cosechadas en arboles tratados con los químicos es mayor. 
Tabla 1. Concentración de solubles y dureza de la pulpa

\begin{tabular}{|c|c|c|}
\hline TRATAMIENTOS & GRADOS BRIX & DUREZA $\left(\mathrm{Kg} / \mathrm{cm}^{2}\right)$ \\
\hline AJO & $15.09^{a}$ & $6.81^{a}$ \\
\hline ERGER @ & $15.29^{a}$ & $6.23^{a}$ \\
\hline REVENT (R) & $14.63^{a}$ & $6.08^{a}$ \\
\hline CONTROL & $14 .^{a}$ & $6.61^{a}$ \\
\hline
\end{tabular}

En la tabla 1 se puede observar que los azucares presentes en las manzanas y la dureza de la pulpa no muestran diferencias significativas (p¿0.05). Sim embargo se puede observar una ligera superioridad en la dureza en los frutos que se trataron con el ajo respecto a las otros compensadores.

Tabla 2. Índices de calidad

\begin{tabular}{cccc}
\hline TRATAMIENTOS & Calibre polar & Calibre ecuatorial & $\mathbf{( \% )}$ de coloración \\
\hline AJO & $5.10^{b}$ & $5.69^{a}$ & $74.58^{a}$ \\
ERGER @ & $5.72^{c}$ & $6.44^{b}$ & $63.33^{b}$ \\
REVENT @ & $5.25^{b}$ & $6.28^{b}$ & $58.33^{b}$ \\
CONTROL & $4.59^{a}$ & $5.61^{a}$ & $59.34^{b}$ \\
\hline
\end{tabular}

En la tabla 2 se observa que el mayor promedio de calibre (tamaño) de fruto fue el tratamiento con Erger, sin embargo, el $74.58 \%$ de coloración fue del ajo (pi0.05).

\section{Conclusiones}

Con base en los resultados se puede concluir que el ajo puede funcionar como compensador de frio en el manzano Red deliciuos, y de mejora la calidad estética del fruto y la dureza de la pulpa, sin embargo, es menos productivo en comparación con los tratamientos de Erger $(\mathrm{R})(16.3 \%)$ y Revent (R) $(20.2 \%)$. Es recomendable seguir investigando el ajo como compensador de frio orgánico con otros ingredientes (aceite invernal) y en situaciones de falta de recursos de hídricos y económicos.

\section{Referencias bibliográficas}

Bomcam, V. E., 2003. Guía para el compostaje y manejo. Convenio Andrés bello. Bogotá. p 22 . ISBN 958-698-103-7

El-Hage, N., Hattam, C., 2003. Agricultura orgánica, ambiente y seguridad alimentaria. Roma: Organización de las Naciones Unidas para la Agricultura y la Alimentación, p.3. ISBN
92-5-304819-0. https://doi.org/10.18356/a48b842bes

Feicán Mejía, C., Encalada A., C., Larriva C., W., y Calle Pérez, G. (1998). El cultivo del manzano para las zonas altas del Austro ecuatoriano. Cuenca, Ecuador: INIAP, Estación Experimental Chuquipta, Programa de Fruticultura. P. 13.

García, L., Martínez, R., Ortega, S., Castro B., 2010. Componentes químicos y su relación con las actividades biológicas de algunos extractos vegetales. Facultad de ciencias biológicas, UJED, Unidad Regional Universitaria de Zonas Áridas-UACh. 12 pag. https://doi.org/10.5154/r.rchsza.2013.03.01

Infoagro., 2007. Comsultado 06 de agosto de 2018. http://www.infoagro.com/frutas/frutas_tradi cionales/manzana.htm

Instituto Nacional de Estadística, Geografia e Informática, 1998. Nuevo Ideal. Estado de Durango. Cuaderno Estadístico Municipal. ISBN 970-13-2008-5.

Martínez-Valenzuela, C., Gómez-Arroyo, S., 2007. Riesgo gonotoxico por exposición a plaguicidas a trabajadores agrícolas. Revista Internacional de 
Contaminación Ambiental, vol. 23, núm. https://doi.org/10.35197/rx.08.03.e2.2012.03.en 4. Universidad Nacional Autónoma de México. Distrito Federal, México. P. 17. https://doi.org/10.20937/rica.2018.34.04.00

Nava-Pérez, E., García-Gutiérrez, C. Olivares, S. E., 1995. Paquete Estadístico de Diseños Experimentales. Facultad de Agronomía, Camacho-Báez, J. \& Vázquez-Montoya, E.

(2012). Bioplaguicidas: Una opción para el Steel G. R., Torrie H. J., 1986. Bioestadística: control biológico de plagas. Ra Ximhail, vol. Principios y Procedimientos. Editorial 8, núm. 3. Sonora, México. 17-29 pp. McGRAWHILL. ISBN 968-451-495-6.

La Revista Ingeniería y Región cuenta con la Licencia Creative Commons Atribución (BY), No Comercial (NC) y Compartir Igual (SA) 